\title{
Perfil de utilização e custos de carbapenêmicos em uma unidade de terapia intensiva de um hospital público do Distrito Federal - Brasil
}

\author{
Use profile and costs of carbapenemics in an intensive care unit in Distrito Federal - Brazil \\ Perfil de uso y costos de carbapenémicos en una unidad de cuidados intensivos del Distrito Federal \\ - Brasil
}

Recebido: 12/10/2021 | Revisado: 22/10/2021 | Aceito: 24/10/2021 | Publicado: 27/10/2021

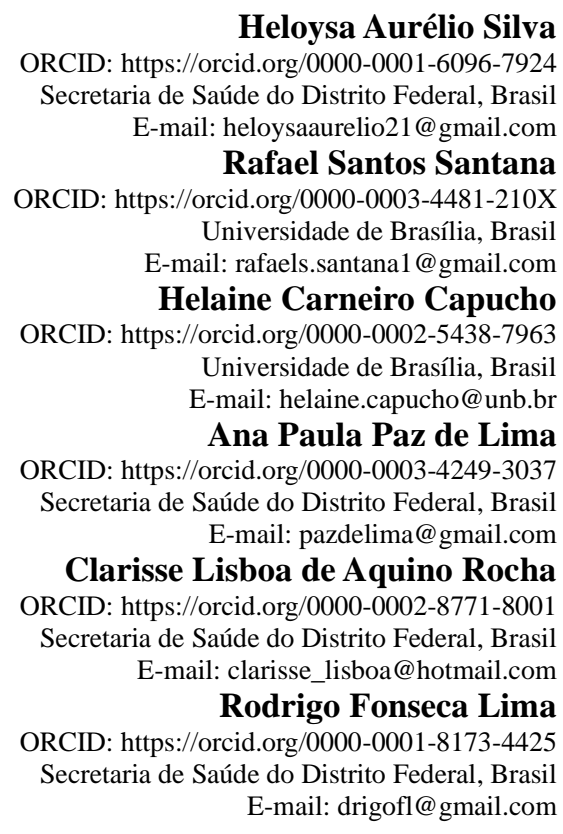

\section{Resumo}

Atualmente o contexto de resistência microbiana tem exigido gerenciamento do uso de antimicrobianos, especialmente no âmbito hospitalar. Assim, o objetivo desse trabalho foi analisar o perfil de utilização de carbapenêmicos na Unidade de Terapia Intensiva (UTI) de um hospital público brasileiro e os custos associados. Para tal, foi realizado um estudo observacional retrospectivo, de análise documental, que avaliou prontuários, prescrições e laudos microbiológicos de pacientes que utilizaram carbapenêmicos em 2019. Foram analisados dados de 25 pacientes com mediana de idade igual a 65 anos e de tempo de internação de 24 dias. Resultaram 36 análises do uso dos carbapenêmicos: $19(57,8 \%)$ remetiam ao uso do meropenem e $17(47,2 \%)$ ao uso de ertapenem. A mediana de dias de uso do meropenem foi de 11 e a do ertapenem foi de sete. A DDD/1000 pacientes-dia do meropenem teve mediana mensal de 115,5g (menor quando utilizado com o ertapenem) e a do ertapenem foi de 50,7g. O gasto anual total foi de $\mathrm{R} \$ 16.652,00$ para o meropenem e de $\mathrm{R} \$ 41.072,00$ para o ertapenem. Foram realizados 60 laudos microbiológicos e os microrganismos mais frequentes foram Klebsiella pneumoniae $(\mathrm{n}=18 ; 30,0 \%)$ e Acinetobacter baumannii complex/haemolyticus $(\mathrm{n}=14 ; 23,3 \%)$; a taxa de sensibilidade geral foi 18,3\% para o meropenem e $13,6 \%$ para o ertapenem. Os dados sugerem a necessidade do gerenciamento no uso de carbapenêmicos considerando aspectos do paciente e do agente infeccioso com potencial impacto positivo nos desfechos clínicos e econômicos. Para isso, propostas de uso racional e seguro de antimicrobianos devem ser implementadas ressaltando a importância de cada profissional no contexto da equipe multidisciplinar.

Palavras-chave: Farmacoepidemiologia; Gestão de antimicrobianos; Assistência hospitalar.

\begin{abstract}
Currently, the context of microbial resistance has required management of the use of antimicrobials, especially in the hospital environment. Thus, the aim of this study was to analyze the profile of carbapenems use in the Intensive Care Unit (ICU) of a Brazilian public hospital and the associated costs. To this end, a retrospective observational study of document analysis was carried out, which evaluated medical records, prescriptions and microbiological reports of
\end{abstract}


patients who used carbapenems in 2019. Data from 25 patients with a median age of 65 years and length of hospital stay were 24 days. There were 36 analyzes of the use of carbapenems: 19 (57.8\%) referred to the use of meropenem and $17(47.2 \%)$ to the use of ertapenem. The median number of days of use of meropenem was 11 and that of ertapenem was seven. The DDD/1000 patient-days of meropenem had a monthly median of $115.5 \mathrm{~g}$ (lower when used with ertapenem) and that of ertapenem was $50.7 \mathrm{~g}$. The total annual expenditure was $\mathrm{R} \$ 16,652.00$ for meropenem and $\mathrm{R} \$ 41,072.00$ for ertapenem. Sixty microbiological reports were carried out and the most frequent microorganisms were Klebsiella pneumoniae $(\mathrm{n}=18 ; 30.0 \%)$ and Acinetobacter baumannii complex/haemolyticus $(\mathrm{n}=14 ; 23.3 \%)$; the overall sensitivity rate was $18.3 \%$ for meropenem and $13.6 \%$ for ertapenem. The data suggest the need to manage the use of carbapenems considering aspects of the patient and the infectious agent with a potential positive impact on clinical and economic outcomes. For this, proposals for the rational and safe use of antimicrobials must be implemented, emphasizing the importance of each professional in the context of the multidisciplinary team.

Keywords: Pharmacoepidemiology; Antimicrobial stewardship; Hospital care.

\section{Resumen}

Actualmente, el contexto de la resistencia microbiana ha requerido el manejo del uso de antimicrobianos, especialmente en el ámbito hospitalario. Así, el objetivo de este estudio fue analizar el perfil de uso de carbapenémicos en la Unidad de Cuidados Intensivos (UCI) de un hospital público brasileño y los costos asociados. Para ello, se realizó un estudio observacional retrospectivo de análisis documental, que evaluó historias clínicas, prescripciones e informes microbiológicos de pacientes que utilizaron carbapenémicos en 2019. Se analizaron datos de 25 pacientes con una mediana de edad de 65 años y tiempo de estancia hospitalaria de 24 días. Se realizaron 36 análisis del uso de carbapenémicos: $19(57,8 \%)$ se refirieron al uso de meropenem y 17 (47,2\%) al uso de ertapenem. La mediana de días de uso de meropenem fue de 11 y la de ertapenem fue de siete. La DDD/1000 pacientes-día de meropenem tuvo una mediana mensual de 115,5 g (menor cuando se usó con ertapenem) y la de ertapenem fue de $50,7 \mathrm{~g}$. El gasto total anual fue de $\mathrm{R} \$ 16.652,00$ para meropenem y $\mathrm{R} \$ 41.072,00$ para ertapenem. Se realizaron 60 informes microbiológicos y los microorganismos más frecuentes fueron Klebsiella pneumoniae $(\mathrm{n}=18 ; 30,0 \%)$ y Acinetobacter baumannii complex/haemolyticus $(\mathrm{n}=14 ; 23,3 \%)$; la tasa de sensibilidad global fue del 18,3\% para meropenem y del 13,6\% para ertapenem. Los datos sugieren la necesidad de manejar el uso de carbapenémicos considerando aspectos del paciente y del agente infeccioso con un potencial impacto positivo en los resultados clínicos y económicos. Para ello, se deben implementar propuestas para el uso racional y seguro de antimicrobianos, enfatizando la importancia de cada profesional en el contexto del equipo multidisciplinario.

Palabras clave: Farmacoepidemiología; Programas de Optimización del uso de los antimicrobianos; Atención hospitalaria.

\section{Introdução}

O uso racional de antimicrobianos (ATM) tem como princípio básico a determinação do agente etiológico e da sua susceptibilidade aos ATM. Além dos fatores relativos aos agentes infectantes, a escolha do ATM deve estar fundamentada no conhecimento de fatores peculiares ao hospedeiro e dos aspectos farmacológicos básicos (farmacocinética, farmacodinâmica) do ATM a ser utilizado (Abdul-Aziz et al., 2020; Brasil, 2017). Os resultados das culturas e antibiogramas fornecem informações importantes sobre o perfil microbiológico de resistência aos ATM nos diferentes setores do hospital. A escolha adequada do ATM é crítica para o sucesso do tratamento, principalmente nas infecções graves (Micek et al., 2010).

Quando ocorrem dificuldades nessa logística assistencial e laboratorial, há consequências clínicas decorrentes do uso indiscriminado de ATM, como a resistência bacteriana, que é considerada um grave problema de saúde pública (Brasil, 2017), sobretudo nos hospitais onde a terapia com ATM é rotineira. O uso inadvertido de ATM pode acarretar em emergência de cepas bacterianas multirresistentes, que podem ser propagadas caso as medidas de precaução não sejam instituídas, afetando tanto o indivíduo como o ambiente no qual está inserido, elevando os custos nos serviços de saúde e os riscos de reações adversas a medicamentos (Baroni et al., 2017; Brasil, 2017). Esse contexto remete à discussão sobre a racionalidade do uso de ATM que, no Brasil, se refletiu na proposta da Agência Nacional de Vigilância Sanitária (ANVISA) de Diretriz Nacional para Elaboração de Programa de Gerenciamento do Uso de Antimicrobianos em Serviços de Saúde em 2017 pública (Brasil, 2017).

Infecções causadas por bactérias multirresistentes são mais difíceis de tratar, envolvem intervenções mais caras e frequentemente com mais efeitos adversos, aumentam o tempo de internação e têm maior mortalidade relacionada (Alp \& Damani, 2015; Wagner et al., 2020). O aumento da morbidade e mortalidade associada à resistência aos ATM é de particular 
preocupação em populações com estado de saúde comprometido, como pacientes em unidades de terapia intensiva (UTI) (Abdul-Aziz et al., 2020; Baroni et al., 2017; Kollef et al., 2017). Reforça-se, então, a importância de racionalizar a prescrição antimicrobiana desde a atenção primária, até o uso em UTI.

O processo de uso do ATM é multifacetado e requer uma abordagem multidisciplinar perpassando por sua escolha, definição de dose, via de administração e duração de tratamento, com o objetivo final de otimizar os resultados dos pacientes, incluindo uma redução antecipada nas taxas de resistência (Chatzopoulou \& Reynolds, 2020). A tarefa de escolha, portanto, destes medicamentos torna-se cada vez mais complexa (Abdul-Aziz et al., 2020; Paiva et al., 2020).

Considerando o relatório da Organização Mundial da Saúde (OMS) de 2020, há uma linha de pesquisa quase estática, com apenas alguns ATM sendo aprovados por agências regulatórias nos últimos anos (Organização Mundial da Saúde, 2020). A maioria desses agentes oferece benefício clínico limitado em relação aos tratamentos existentes, com 82\% dos ATM aprovados recentemente sendo derivados de classes de antibióticos. Portanto, é esperado um rápido surgimento de resistência a esses novos agentes, limitando a sua utilidade e não solucionando a necessidade de novas alternativas terapêuticas (Alp \& Damani, 2015; Lima et al., 2011).

Dentre as alternativas disponíveis, há destaque para os carbapenêmicos. Sua estabilidade contra a hidrólise pela maioria das beta-lactamases torna o grupo uma importante alternativa no tratamento de infecções graves, incluindo as causadas por bactérias multirresistentes (Lima et al., 2011; McCann et al., 2018; Wagner et al., 2020). Esse grupo de ATM é ativo contra as principais bactérias gram-positivas (exceto Staphylococcus aureus resistente à meticilina, Staphylococcus epidermidis, Enterococcus faecium e Enterococcus faecalis - imipenem geralmente possui ação contra este último) e gramnegativas (exceto Stenotrophomonas maltophilia, que possui resistência intrínseca aos carbapenêmicos e Pseudomonas aeruginosa e Acinetobacter baumannii, com resistência intrínseca ao ertapenem) e espécies anaeróbicas (Patrier \& Timsit, 2020).

Dada sua importância clínica, o gerenciamento do emprego dos ATM é imprescindível. A literatura aponta que, com a restrição e revisão prospectivas destes, há tendência de redução na incidência de bactérias gram-negativas multirresistentes (Abdallah et al., 2019). No entanto, a prevalência de bactérias gram-negativas resistentes a carbapenêmicos tem aumentado significativamente na última década (Brasil, 2017).

As infecções decorrentes de agentes resistentes a carbapenêmicos representam um grave problema de saúde pública. Ações de controle e prevenção de infecções relacionadas à assistência de saúde, gerenciamento de ATM e a correta identificação dos microrganismos em tempo hábil, têm a finalidade de reduzir a resistência a esse grupo de ATM em ambientes de saúde (Lima et al., 2011; McCann et al., 2018). Nesse contexto, o objetivo deste trabalho foi analisar o perfil de utilização de carbapenêmicos na UTI de um hospital público do Distrito Federal (DF) e os custos associados.

\section{Metodologia}

Trata-se de estudo observacional retrospectivo, realizado na UTI de um hospital público do Distrito Federal de médio porte. Analisaram-se prontuários, prescrições e laudos microbiológicos de pacientes internados e que tiveram prescrição de carbapenêmicos no ano de 2019 (uso do ATM $\geq 24 \mathrm{~h}$ ) com diagnóstico de infecção documentada microbiologicamente, bem como perfil de sensibilidade do agente causador mediante análise de laudos microbiológicos. Foram excluídos pacientes com indisponibilidade de alguns dos dados necessários para o alcance do objetivo do estudo.

As informações coletadas envolveram variáveis relacionadas aos pacientes (idade, sexo, peso, altura, creatinina sérica, Terapia Renal Substitutiva (TRS), diagnóstico (de acordo com a Classificação Internacional de Doenças), tempo de internação na unidade e desfechos clínicos) e aos ATM prescritos propriamente ditos (medicamento, concentração, forma farmacêutica, 
dose, posologia, duração de tratamento, orientações de administração e custo) referentes ao período de internação do paciente na UTI.

Ademais, foram analisados os laudos microbiológicos provenientes do equipamento utilizado pelo laboratório do hospital (MicroScan WalkAway 96 Plus®). Os relatórios corresponderam a laudos de cultura (hemocultura, urocultura e cultura de aspirado traqueal), bem como perfis de sensibilidade e resistência dos microrganismos aos ATM. Os laudos referentes às Concentração Inibitória Mínima (CIM) por microrganismos conforme ATM também foram obtidos. Quando o mesmo paciente remetia a diferentes laudos referentes ao mesmo microrganismo provenientes do mesmo produto, foram analisados os perfis de sensibilidade e resistência e CIM para confirmação considerando a possibilidade de ser o mesmo agente infeccioso, mas com perfil de suscetibilidade diferente.

Os dados coletados permitiram a construção de indicadores do processo de utilização dos ATM, indicadores de desfecho/resultado clínicos e financeiros conforme especificado na Diretriz Nacional para Elaboração de Programa de Gerenciamento do Uso de Antimicrobianos em Serviços de Saúde (Brasil, 2017).

O indicador de processos referente à utilização dos carbapenêmicos utilizados foi Dose Diária Definida (DDD)/ 1000 pacientes-dia (Organização Mundial da Saúde, 2021). Dentre os indicadores de desfecho/resultado, foram utilizados o de resistência/sensibilidade microbiana (mediante cálculo da taxa de sensibilidade aos carbapenêmicos) e frequência de infecção por bactérias multirresistentes de destaque (Brasil, 2017). Além disso, foram analisados dados clínicos (tempo de internação e desfechos clínicos) e calculado o custo da terapia, além de verificadas orientações para a administração dos ATM em questão (Brasil, 2017).

O formulário informatizado permitiu a construção de um banco no Excel® para armazenamento e análise dos dados. A descrição das variáveis categóricas foi realizada por meio do cálculo das frequências absolutas e relativas e as variáveis contínuas foram reportadas pela mediana por se tratar de uma medida central mais robusta menos afetada por valores muito diferentes dos demais.

Cada período de uso dos medicamentos foi considerado uma unidade analítica, de modo que um mesmo paciente pode ter resultado em mais de uma análise dado que pode ter utilizado o carbapenêmicos em períodos não consecutivos ao longo da internação. Ademais, quando possível, os resultados foram apresentados de maneira comparativa considerando os carbapenêmicos utilizados (monoterapia com meropenem ou duplo carbapenêmico com utilização conjunta de meropenem e ertapenem).

O estudo faz parte de um projeto mais amplo com propósito de avaliar o uso de ATM no hospital em questão e foi aprovado pelo comitê de ética da Fundação de Ensino e Pesquisa em Ciências da Saúde (FEPECS/ SES/ DF) (CAAE 30205920.5.0000.5553).

\section{Resultados}

Foram analisados dados de 25 pacientes de um total de 52 que tiveram prescrição de carbapenêmicos ao longo do ano de 2019. Os dados referentes aos pacientes em termos epidemiológicos e clínicos estão apresentados na Tabela 1. 
Tabela 1: Dados epidemiológicos e clínicos dos pacientes que tiveram prescrição de carbapenêmicos na UTI no ano de 2019.

\begin{tabular}{|c|c|c|c|}
\hline \multirow[b]{2}{*}{ Variáveis* } & \multirow[b]{2}{*}{ Total $(n=25)$} & \multicolumn{2}{|c|}{ Descrição por carbapenêmicos } \\
\hline & & $\begin{array}{l}\text { Meropenem } \\
(\mathbf{n}=11)\end{array}$ & $\begin{array}{l}\text { Meropenem + ertapenem } \\
(\mathbf{n}=14)\end{array}$ \\
\hline Idade (mediana) (anos) & 65 & 65 & 72,5 \\
\hline Homens (n [\%]) & $14(56,0)$ & $8(72,7)$ & $6(42,9)$ \\
\hline \multicolumn{4}{|l|}{ Diagnóstico à admissão (n [\%]) } \\
\hline Doenças do aparelho geniturinário & $4(16,0)$ & $2(18,2)$ & $2(14,3)$ \\
\hline Doenças do sistema digestivo & $4(16,0)$ & $2(18,2)$ & $2(14,3)$ \\
\hline $\begin{array}{l}\text { Sintomas, sinais e achados anormais de exames clínicos e de } \\
\text { laboratório*** }\end{array}$ & $4(16,0)$ & $1(9,1)$ & $3(21,4)$ \\
\hline Doenças do sistema respiratório & $3(12,0)$ & $0(0,0)$ & $3(21,4)$ \\
\hline Doenças do sistema circulatório & $2(8,0)$ & $0(0,0)$ & $2(14,3)$ \\
\hline Doenças do sistema osteomuscular e do tecido conjuntivo & $2(8,0)$ & $2(18,2)$ & $0(0,0)$ \\
\hline Doenças infecciosas & $2(8,0)$ & $2(18,2)$ & $0(0,0)$ \\
\hline Neoplasias (tumores) & $2(2,0)$ & $2(18,2)$ & $0(0,0)$ \\
\hline Doenças endócrinas, nutricionais e metabólicas & $1(4,0)$ & $0(0,0)$ & $1(7,1)$ \\
\hline Outras & $1(4,0)$ & $0(0,0)$ & $1(7,1)$ \\
\hline Tempo de internação (mediana) (dias) & 24 & 11 & 34 \\
\hline Peso (mediana) (quilogramas) & 68,0 & 70,6 & 66,0 \\
\hline Creatinina sérica (mediana) $(\mathrm{mg} / \mathrm{dL})$ & 2,1 & 2,1 & 2,0 \\
\hline Clearence de creatinina (mediana) $(\mathrm{mL} / \mathrm{min})$ & 32,1 & 32,1 & 31,8 \\
\hline Terapia Renal Substitutiva (n [\%]) & $19(76,0)$ & $8(72,7)$ & $11(78,6)$ \\
\hline \multicolumn{4}{|l|}{ Desfechos clínicos (n [\%]) } \\
\hline Alta & $3(12,0)$ & $2(18,2)$ & $1(7,1)$ \\
\hline Óbito por choque séptico & $17(68,0)$ & $7(63,6)$ & $10(71,4)$ \\
\hline Óbito por causas não infecciosas & $5(20,0)$ & $2(18,2)$ & $3(21,4)$ \\
\hline
\end{tabular}

*\% em relação ao n da coluna. **não classificados em outra parte.

Fonte: Dados da pesquisa (2019).

Dos 25 pacientes, oito (32,2\%) utilizaram carbapenêmicos em períodos não consecutivos ao longo da internação, resultando em 36 análises. Dessas 36, 19 (57,8\%) remetiam ao uso apenas do meropenem e 17 (47,2\%) remetiam ao uso de meropenem e ertapenem de maneira concomitante (duplo carbapenêmico). A mediana de dias de uso do meropenem foi de 11 e a do ertapenem foi de sete.

O total de pacientes-dia na UTI foi de 2339, variando de 147 a 227 ao longo do ano de 2019. A DDD/1000 pacientesdia do meropenem variou de $21 \mathrm{~g}$ a $308 \mathrm{~g}$ ao longo do ano, com mediana mensal igual a $115,5 \mathrm{~g}$, sendo menor quando usado junto com o ertapenem. O ertapenem teve DDD/1000 pacientes-dia variando de $13 \mathrm{~g}$ a $190 \mathrm{~g}$ com mediana mensal igual a $50,7 \mathrm{~g}$. O custo unitário foi de $\mathrm{R} \$ 18,00$ para o meropenem e $\mathrm{R} \$ 302,00$ para o ertapenem, resultando em um custo anual total de $\mathrm{R} \$ 16.652,00$ e $\mathrm{R} \$ 41.072,00$, respectivamente. O custo total referente ao meropenem quando usado isoladamente foi de $\mathrm{R} \$ 10.773,20$ ( $\mathrm{R} \$ 979,40 /$ paciente), enquanto o custo total referente ao uso do duplo carbapenêmico foi de $\mathrm{R} \$ 46.950,80$ (R $\$ 3.353,60 /$ paciente). Os dados referentes ao consumo dos carbapenêmicos estão apresentados na forma de indicadores na Tabela 2 . 
Research, Society and Development, v. 10, n. 14, e99101421691, 2021

(CC BY 4.0) | ISSN 2525-3409 | DOI: http://dx.doi.org/10.33448/rsd-v10i14.21691

Tabela 2: Indicadores de processo e financeiros referentes à utilização dos carbapenêmicos na UTI no ano de 2019.

\begin{tabular}{|c|c|c|c|c|c|c|c|c|c|c|c|c|c|}
\hline \multirow{3}{*}{ Mês } & \multirow{3}{*}{ Pet/dia } & \multicolumn{9}{|c|}{ Meropenem } & \multirow{2}{*}{\multicolumn{3}{|c|}{ Ertapenem }} \\
\hline & & \multicolumn{3}{|c|}{ Prescrito isoladamente } & \multicolumn{3}{|c|}{ Prescrito com ertapenem } & \multicolumn{3}{|c|}{ Total } & & & \\
\hline & & $\begin{array}{c}\text { Consumo } \\
(\mathrm{g})\end{array}$ & DDD (g) & $\begin{array}{c}\text { Custo } \\
\text { (R\$) }\end{array}$ & $\begin{array}{c}\text { Consumo } \\
\text { (g) }\end{array}$ & DDD (g) & $\begin{array}{c}\text { Custo } \\
\text { (R\$) }\end{array}$ & $\begin{array}{c}\text { Consumo } \\
\text { (g) }\end{array}$ & DDD (g) & $\begin{array}{c}\text { Custo } \\
\text { (R\$) }\end{array}$ & $\begin{array}{c}\text { Consumo } \\
\text { (g) }\end{array}$ & DDD (g) & $\begin{array}{c}\text { Custo } \\
(\mathbf{R} \$)\end{array}$ \\
\hline Fevereiro & 147 & 81 & 184 & $1.490,40$ & 6 & 14 & 110,40 & 87 & 197 & $1.600,80$ & 28 & 190 & $8.456,00$ \\
\hline Março & 171 & 28 & 55 & 515,20 & 0 & 0 & 0,00 & 28 & 55 & 515,20 & 7 & 41 & $2.114,00$ \\
\hline Abril & 156 & 29 & 62 & 533,60 & 84,0 & 179 & $1.545,60$ & 113 & 241 & $2.079,20$ & 7 & 45 & $2.114,00$ \\
\hline Maio & 189 & 12 & 21 & 220,80 & 0 & 0 & 0,00 & 12 & 21 & 220,80 & 8 & 42 & $2.416,00$ \\
\hline Junho & 210 & 21 & 33 & 386,40 & 9 & 14 & 165,60 & 30 & 48 & 552,00 & 3 & 14 & 906,00 \\
\hline Julho & 218 & 24 & 37 & 441,60 & 20 & 31 & 368,00 & 44 & 67 & 809,60 & 4 & 18 & $1.208,00$ \\
\hline Agosto & 225 & 34 & 50 & 625,60 & 54 & 80 & 993,60 & 88 & 130 & $1.619,20$ & 16 & 71 & $4.832,00$ \\
\hline Setembro & 199 & 159 & 266 & $2.925,60$ & 6,5 & 11 & 119,60 & 165,5 & 277 & $3.045,20$ & 15 & 75 & $4.530,00$ \\
\hline Outubro & 227 & 31 & 46 & 570,40 & 6 & 9 & 110,40 & 37 & 54 & 680,80 & 3 & 13 & 906,00 \\
\hline Novembro & 212 & 28 & 44 & 515,20 & 54 & 85 & 993,60 & 82 & 129 & $1.508,80$ & 12 & 57 & $3.624,00$ \\
\hline Dezembro & 222 & 68 & 102 & $1.251,20$ & 0 & 0 & 0,00 & 68 & 102 & $1.251,20$ & 21 & 95 & $6.342,00$ \\
\hline Total & 2339 & 586 & 83 & $10.773,20$ & 319,5 & 46 & $5.878,80$ & 905 & 1630 & $16.652,00$ & 136 & 736 & $41.072,00$ \\
\hline
\end{tabular}

DDD: Dose Diária Definida; Pct: paciente; Tto: tratamento.

Fonte: Dados da pesquisa (2019). 
Nem todas as prescrições dos carbapenêmicos apresentavam registro de orientações para administração do medicamento. Dos pacientes que tiveram prescrição de meropenem (com ou sem prescrição conjunta ao ertapenem), nove $(36,0 \%)$ tiveram pelo menos um dia de prescrição sem orientação quanto ao tempo de infusão, sendo que três deles tiveram suas prescrições sem essa informação ao longo de todo o tempo de tratamento.

Quanto ao ertapenem, todas as prescrições apresentaram a informação de tempo de infusão, no entanto, para cinco pacientes (35,7\% dos 14 que tiveram prescrição de ertapenem de maneira conjunta ao meropenem), não houve definição do horário para sua administração com correspondência à administração do outro carbapenêmico (em um deles também não havia orientação para administração do meropenem; os cinco faleceram por choque séptico).

Os 25 pacientes remeteram a 60 laudos microbiológicos com referência a microrganismos distintos, seja em termos qualitativos seja quanto à suscetibilidade, sendo a maioria deles resultantes da análise de aspirado traqueal ( $\mathrm{n}=36 ; 60,0 \%)$, seguido de sangue $(\mathrm{n}=10 ; 16,7 \%)$ e de urina $(\mathrm{n}=4 ; 6,7 \%)$.

Os microrganismos mais frequentes foram Klebsiella pneumoniae $(\mathrm{n}=18 ; 30,0 \%$ - três delas identificadas como produtoras de beta-lactamases de espectro estendido (ESBL) pelo laudo), Acinetobacter baumannii complex/haemolyticus $(\mathrm{n}=14 ; 23,3 \%)$ e Pseudomonas aeruginosa $(\mathrm{n}=9 ; 15,0 \%)$ seguidos pela Stenotrophomonas maltophilia $(\mathrm{n}=8 ; 13,3)($ tabela 3$)$. A taxa de sensibilidade geral foi 18,3\% para o meropenem e 13,6\% para o ertapenem com variação entre os microrganismos, excluindo-se as resistências intrínsecas, conforme apresentado na tabela 3.

Tabela 3: Perfil microbiológico dos agentes causadores de infecção dos pacientes que tiveram prescrição de carbapenêmicos na UTI no ano de 2019.

\begin{tabular}{|c|c|c|c|c|c|}
\hline \multirow[b]{2}{*}{ Microrganismo } & \multirow{2}{*}{$\begin{array}{c}\begin{array}{c}\mathbf{N} \text { de isolados } \\
(\%)\end{array} \\
\begin{array}{c}\text { Todos os } \\
\text { pacientes } \\
(\mathrm{n}=25 ; 36 \\
\text { análises) }\end{array}\end{array}$} & \multicolumn{2}{|c|}{$\begin{array}{c}\mathrm{N} \text { de isolados por tipo de tratamento } \\
(\%)\end{array}$} & \multicolumn{2}{|c|}{$\begin{array}{c}\text { N de isolados susceptíveis } \\
(\%) \text { ao } \\
\end{array}$} \\
\hline & & $\begin{array}{c}\text { Pacientes em uso } \\
\text { somente de } \\
\text { meropenem } \\
(n=11 ; 19 \\
\text { análises }) \\
\end{array}$ & $\begin{array}{c}\text { Pacientes em uso } \\
\text { de meropenem + } \\
\text { ertapenem } \\
(n=14 ; 17 \\
\text { análises) } \\
\end{array}$ & Meropenem & Ertapenem \\
\hline Klebsiella pneumoniae & $\begin{array}{c}18(30,0)^{3} \\
\text { ESBL+ }\end{array}$ & $8(44,4)$ & $10(55,6)$ & $2(11,1)$ & $2(11,1)$ \\
\hline $\begin{array}{l}\text { Acinetobacter baumannii } \\
\text { complex/haemolyticus }\end{array}$ & $14(23,3)$ & $7(50,0)$ & $7(50,0)$ & $0(0,0)$ & $0(0,0)^{*}$ \\
\hline Pseudomonas aeruginosa & $9(15,0)$ & $6(66,7)$ & $3(33,3)$ & $2(22,2)$ & $0(0,0)^{*}$ \\
\hline Stenotrophomonas maltophilia & $8(13,3)$ & $1(12,5)$ & $7(87,5)$ & $0(0,0) *$ & $0(0,0) *$ \\
\hline Serratia marcescens & $4(6,7)^{1 \mathrm{ESBL}+}$ & $2(50,0)$ & $2(50,0)$ & $3(75,0)$ & $3(75,0)$ \\
\hline Enterobacter cloacae & $2(3,3)$ & $1(50,0)$ & $1(50,0)$ & $2(100,0)$ & $1(50,0)$ \\
\hline Escherichia coli & $2(3,3)$ & $1(50,0)$ & $1(50,0)$ & $2(100,0)$ & $2(100,0)$ \\
\hline Chryseobacterium indologenes & $1(1,7)$ & $1(100,0)$ & $0(0,0)$ & $0(0,0)$ & Não testado \\
\hline Proteus vulgaris. & $1(1,7)$ & $1(100,0)$ & $0(0,0)$ & $0(0,0)$ & $0(0,0)$ \\
\hline Pseudomonas fluorescens/putida & $1(1,7)$ & $0(0,0)$ & $1(100,0)$ & $0(0,0)$ & $0(0,0)^{*}$ \\
\hline Total & $60(100,0)$ & $28(46,7)$ & $32(53,3)$ & $11(18,3)$ & $8(13,6) * *$ \\
\hline
\end{tabular}

ESBL+: bactéria produtora de beta-lactamases de espectro estendido. *Resistência intrínseca. **Desconsiderando a isolado não testado (Chryseobacterium indologenes).

Fonte: dados da pesquisa (2019).

\section{Discussão}

O uso de carbapenêmicos na UTI do hospital em questão foi, de maneira geral, em pacientes críticos essencialmente idosos e com disfunção renal importante cujas infecções, em sua maioria, eram causadas por bactérias gram-negativas multirresistentes. Em termos de perfil epidemiológico, os resultados vão de acordo com outros realizados em contextos semelhantes (Alyahawi et al., 2020; Baroni et al., 2017; Codjoe \& Donkor, 2018; Coêlho et al., 2020; Souza et al., 2021), 
chamando atenção para a mediana de idade alta, que pode ser reflexo do processo de transição demográfica atualmente evidenciado no Brasil.

Cabe ressaltar a elevada proporção de desfechos clínicos desfavoráveis, superior a outros estudos (Baroni et al., 2017; Silva et al., 2011; Wagner et al., 2020). Entretanto, as características clínicas dos pacientes (repercutindo inclusive na mediana de tempo de internação na UTI superior à achada em outras pesquisas (Araujo et al., 2013; Baroni et al., 2017)) podem justificar esse resultado. A frequência de óbito por choque séptico chama atenção, mesmo não havendo especificação de infecção no diagnóstico à admissão. Desta forma, cabe discussão acerca do papel das Infecções Relacionadas à Assistência à Saúde (IRAS) neste contexto, especialmente considerando o perfil microbiológico evidenciado.

O âmbito assistencial complexo em questão associado ao perfil do paciente crítico propicia um contexto favorável à ocorrência dessas IRAS (Alp \& Damani, 2015; Coêlho et al., 2020; Khan et al., 2017; Souza et al., 2021) geralmente associadas a bactérias gram-negativas multirresistentes (Alp \& Damani, 2015; Joseph et al., 2015; Khan et al., 2017). A taxa de consumo de antimicrobianos de amplo espectro prescritos de forma inadequada e indiscriminada também está relacionada à emergência de bactérias multirresistentes, frequentemente encontradas em IRAS (Alp \& Damani, 2015; Lima et al., 2011).

Em termos gerais, o perfil microbiológico encontrado está em consonância com o que vem sendo discutido na literatura: há um rápido aumento da prevalência de Enterobactérias abrigando carbapenemases, como as Klebsiella spp. Ademais, Acinetobacter baumannii complex/haemolyticus e Pseudomonas aeruginosa vem sendo referidos como principais agentes infecciosos causadores de IRAS no âmbito da terapia intensiva (Alp \& Damani, 2015; Joseph et al., 2015; Khan et al., 2017; Lima et al., 2011).

A resistência microbiana pode se tornar a principal causa de mortalidade em 2050 (Gajdács, 2019; O’Neill, 2014). Dentre os agentes que são mais preocupantes do ponto de vista clínico e de resistência são os chamados patógenos "ESKAPE" (E: Enterococcus faecium, S: Staphylococcus aureus ou recentemente Stenotrophomonas maltophilia, K: Klebsiella pneumoniae ou recentemente C: Clostridioides difficile, A: Acinetobacter baumannii, P: Pseudomonas aeruginosa, E: Enterobacter spp., ou recentemente Enterobacteriaceae) (Gajdács, 2019). O perfil microbiológico evidenciado neste estudo remeteu a quase todos os microrganismos desse acrônimo.

A incidência de infecções por bacilos gram-negativos multirresistentes está aumentando em todo o mundo e, de forma proporcional, há maior complexidade da terapêutica envolvida, já que muitas vezes restam poucos (ou nenhum) ATM eficaz (Abdallah et al., 2019; Lima et al., 2011; McCann et al., 2018). Além disso, essas infecções geralmente são graves, em pacientes com estado crítico, associadas a internações hospitalares mais longas e alta taxa de mortalidade (Abdallah et al., 2019), o que também foi evidenciado no presente estudo.

O meropenem é um carbapenêmico amplamente utilizado em UTI como terapia empírica em casos de infecções graves e, mesmo após laudo microbiológico, sua prescrição tende a se manter na perspectiva de manutenção da ampla cobertura contra agentes infectantes, o que pode ser plausível considerando a frequência de IRAS nesse contexto (Balkhy et al., 2018; Baroni et al., 2017). Além disso, inadequações estruturais encorajam a prescrição de antimicrobianos em excesso (especialmente de amplo espectro) para cobrir todos os agentes possíveis causadores de infecção, sendo comum a prescrição desse ATM em UTI (Alp \& Damani, 2015; Carvalho et al., 2021; Santos et al., 2020; Souza et al., 2021).

Entretanto, ressalta-se que o uso racional e responsável do meropenem guiado por cultura é que de fato impacta positivamente na assistência em termos de redução de morbi-mortalidade. Assim, a conduta de prescrição do carbapenêmico, no entanto, deve ser discutida no âmbito de um programa de gerenciamento de ATM e considerar a possibilidade de resistência. O perfil de sensibilidade dos microrganismos infectantes encontrados nesse estudo revela essa necessidade.

A DDD/ 1000 pacientes/dia encontrada do meropenem foi superior à encontrada em outros estudos (Furtado et al., 2019; Lobo et al., 2017), mas o perfil epidemiológico e clínico em termos de gravidade dos pacientes pode justificar isso. 
Outros indicadores de processo devem ser considerados para embasar comparações sobre uso de medicamentos, apesar da DDD ser um método bastante usado nesse sentido (Brasil, 2017; Joseph et al., 2015). É importante referir que a DDD não representa a dose recomendada e que especificidades dos pacientes devem ser consideradas, como idade, peso e função renal do paciente, além de características relacionadas ao microrganismo (Baroni et al., 2017; Brasil, 2017; Joseph et al., 2015; Souza et al., 2021).

Não foi verificada a utilização isolada do ertapenem neste estudo. A hipótese que embasa sua prescrição de forma isolada é o uso preferencial em infecções na qual há suspeita ou confirmação de bactérias produtoras de ESBL, exercendo pressão seletiva para bactérias não fermentadoras resistentes aos carbapenêmicos (Pseudomonas aeruginosa, Acinetobacter baumannii complex/haemolyticus e Stenotrophomonas maltophilia) (Chatzopoulou \& Reynolds, 2020; Lima et al., 2011). Entretanto, o perfil microbiológico dos agentes causadores de infecção na UTI já apresentava uma alta frequência desses isolados não fermentadores, o que pode justificar o uso de ertapenem somente em associação ao meropenem e refletir escolha adequada dos prescritores nestes casos.

Apesar do ertapenem não ser efetivo contra Acinetobacter baumannii e Pseudomonas aeruginosa, ele possui alta afinidade com carbapenemases, o que embasa a proposta do seu uso como carbapenêmico "suicida" na tentativa de aumento da atividade de outro carbapenêmico (o meropenem, no caso desse estudo) (Patrier \& Timsit, 2020). A terapia com duplo carbapenêmico consiste na administração de ertapenem 30 a 60 minutos antes da primeira infusão prolongada (três horas) de meropenem (Patrier \& Timsit, 2020; White et al., 2019).

Houve uma elevada frequência da associação de meropenem e ertapenem, cabendo ressaltar que o DDD/ 1000 pacientes/dia do meropenem quando usado no esquema de duplo carbapenêmico foi menor. Entretanto, o período de internação dos pacientes que usaram duplo carbapenêmico foi maior, com maior frequência de óbito por choque séptico. Os objetivos da associação são, de maneira geral, aumentar sinergicamente o espectro de ação, reduzir risco de mortalidade de pacientes com infecção por bactérias resistentes e evitar o uso de outros ATM visando reduzir risco de aquisição de mecanismos de resistência e diminuir risco (ou piora do quadro) de disfunção renal (Oliva et al., 2017; Pascale et al., 2017; White et al., 2019).

Os efeitos da terapia com duplo carbapenêmico são descritos por vários estudos in vitro apesar de ainda haver pouca literatura que fundamente e discuta desfechos clínicos resultantes dessa associação in vivo (White et al., 2019). Li et al. (2020) em revisão sistemática referem que, devido à resposta clínica semelhante a outras alternativas e menor mortalidade, a terapia de duplo carbapenêmico pode corresponder a uma opção para tratamento de infecções causadas por bactérias gram-negativas resistentes (Li et al., 2020). Entretanto, mais estudos são necessários, principalmente com ensaios clínicos de maior qualidade que abordem desfechos relacionados à efetividade, segurança do paciente e risco de resistência do microrganismo (Li et al., 2020).

Alguns estudos observacionais demonstraram efeitos positivos com o uso de duplo carbapenêmico desde que seguidas as orientações quanto à administração visando otimização da terapia (Oliva et al., 2017; Pascale et al., 2017; Patrier \& Timsit, 2020; White et al., 2019). Entretanto, nem todas as prescrições dos pacientes com duplo carbapenêmico avaliadas no presente estudo apresentavam todas as orientações para administração dos medicamentos, o que evidencia a importância da comunicação entre os membros da equipe assistencial em relação ao processo de uso dos medicamentos no cuidado ao paciente. Soma-se a esse contexto a necessidade de abordagem dos reações adversas relacionadas aos carbapenêmicos e à necessidade de farmacovigilância associada, especialmente considerando que a proposição do duplo carbapenêmico é considerada “off-label” (Patrier \& Timsit, 2020).

É importante referir que a taxa de sensibilidade aos carbapenêmicos foi baixa, o que pode ter influenciado na alta taxa de mortalidade observada já que teoricamente não haveria efeito clínico (com ou sem utilização de duplo carbapenêmico). No entanto, cabe considerar que além dos perfis de sensibilidade, deve-se avaliar o perfil de distribuição do ATM para verificação 
da sua disponibilidade no sítio de infecção considerando CIM desejada para definição da conduta (Brasil, 2017). Ademais, deve-se considerar que o perfil dos pacientes em uso desses ATM, cuja criticidade pode enviesar a análise relacionada à morbimortalidade.

O meropenem, por ser um betalactâmico, tem efeito dependente do tempo de concentração em que está acima da CIM. Desta forma, é importante otimizar seu uso e, de maneira geral, prolongar sua vida clínica reduzindo o surgimento de resistência bacteriana (Abdul-Aziz et al., 2020). Isso, no entanto, é difícil em pacientes críticos já que possuem grande variabilidade farmacocinética (Abdul-Aziz et al., 2020; Roberts et al., 2014), como no caso dos pacientes incluídos neste estudo.

Se essa variabilidade não for considerada para otimização da terapia, pode haver um aumento do risco de falha terapêutica e/ou resistência bacteriana por baixa exposição ao medicamento ou aumento do risco de toxicidade por alta exposição ao medicamento (Abdul-Aziz et al., 2020). Essa otimização envolve a consideração de dados da CIM do agente infectante, dados farmacocinéticos e clínicos dos pacientes para definição da melhor posologia e dose, visando o sucesso terapêutico. Entretanto, nenhum esquema prescrito avaliado considerou essa perspectiva, apresentando-se como uma lacuna importante para intervenções.

Ressalta-se que estudos de utilização de ATM são importantes também do ponto de vista econômico, já que correspondem a um dos grupos de medicamentos que mais responde por gastos hospitalares com implicações importantes para todos os atores envolvidos no processo assistencial (pacientes, gestores e pagadores) (Alyahawi et al., 2020; Rodrigues \& Bertoldi, 2010; Souza et al., 2021). Nesse sentido, cabe destaque aos custos associados aos carbapenêmicos, que estão entre os ATM mais utilizados e com maiores custos associados no contexto de terapias intensivas (Baroni et al., 2017; Santos et al., 2020) exigindo a necessidade de racionalidade no seu uso. Os resultados observados corroboram essa discussão, especialmente considerando o custo consideravelmente mais alto quando da associação dos carbapenêmicos (quase cinco vezes maior). Embora a associação não tenha mostrado diferenças em termos descritivos, o contexto requer uma análise econômica de forma mais apropriada e concernente ao contexto clínico dos pacientes críticos.

Outros fatores além dos relacionados ao uso inadequado de ATM (principalmente os de amplo espectro) podem justificar o aumento da resistência microbiana. Dentre esses fatores cabe destaque à necessidade de um processo de treinamento e educação continuada dos atores envolvidos na assistência ao paciente. Soma-se a esse contexto a perspectiva clínica relacionada à necessidade de reavaliação contínua da necessidade de ATM e da possibilidade de terapia sequencial em uma perspectiva de custo-efetividade, checagem diária dos resultados de culturas, avaliação de dispositivos invasivos, que, dada a rotina assistencial, podem não acontecer de maneira adequada (Alp \& Damani, 2015).

Aspectos gerenciais relacionados à disponibilidade de instalações e dispositivos de higiene das mãos abaixo do que é necessário e ruptura de estoque de equipamentos de proteção individual e/ou produtos para saúde e medicamentos, também devem ser considerados. Quando do desabastecimento de ATM de uso restrito como polimixina B ou tigeciclina, situações inclusive vivenciadas no local de estudo, as opções para tratamento de pacientes críticos com infecções graves diminuem e, quando em abastecimento regular, a alternativa de duplo carbapenêmico se sobressai.

Os resultados observados devem ser analisados sob a ótica de limitações da metodologia utilizada cujo objetivo geral foi conhecer o padrão de prescrição de forma inicial além do perfil microbiológico, sem estabelecer relações causais diretas. A pesquisa seguirá com o intuito de identificar o alcance de objetivos farmacocinéticos do uso dos carbapenêmicos nesses pacientes com base nas suas características mediante cálculos específicos visando individualizar a terapia. Nesse cenário posterior, caberá ainda a verificação de outros ATM usados de forma concomitante aos carbapenêmicos e impactos em termos de efeitos e eventos adversos associados. A longo prazo, esta pesquisa pretende sugerir, em um contexto multidisciplinar, critérios adequados para otimizar o efeito dos ATM em pacientes críticos. 


\section{Conclusão}

Os carbapenêmicos no contexto da UTI do trabalho em tela foram usados por pacientes em sua maioria idosos com doença crítica em grau importante e infecções causadas principalmente por bactérias gram-negativas multirresistentes. Quase metade da amostra utilizou duplo carbapenêmico com custo quase cinco vez maior quando comparada à terapia com meropenem.

Os dados evidenciados remontam à importância e à necessidade do gerenciamento na prescrição de carbapenêmicos visando monitoramento da necessidade do seu uso considerando aspectos do paciente e do perfil microbiológico da unidade em que ele está inserido. A longo prazo, mais desfechos clínicos favoráveis, redução de custos relacionados à assistência e mudança do perfil microbiológico também poderão ser evidenciados.

É necessário que políticas e propostas de uso racional e seguro de ATM sejam implementadas e praticadas por toda a equipe do programa de gerenciamento de uso de ATM envolvida na assistência. Para tal, requer-se protocolos e diretrizes para auxílio na definição da terapia, empírica ou não, de pacientes com infecções graves em unidades de terapia intensiva. Nesse contexto, há exigência de um papel mais ativo do farmacêutico clínico e o apoio da alta administração da instituição visando, em maior espectro, agregar valor, qualidade e segurança ao processo assistencial.

\section{Agradecimentos}

Agradecimentos à Secretaria de Saúde do Distrito Federal pelo apoio estrutural e institucional.

\section{Referências}

Abdallah, M., Badawi, M., Alzaagi, I., Issa, K. N., Rasheed, A., \& Alharthy, A. (2019). Effect of short-term carbapenem restriction on the incidence of nonpseudomonal multi-drug resistant Gram-negative bacilli in an intensive care unit. Journal of Chemotherapy, 31(5), 261-266. https://doi.org/10.1080/1120009X.2019.1601802

Abdul-Aziz, M. H., Alffenaar, J. W. C., Bassetti, M., Bracht, H., Dimopoulos, G., Marriott, D., Neely, M. N., Paiva, J. A., Pea, F., Sjovall, F., Timsit, J. F., Udy, A. A., Wicha, S. G., Zeitlinger, M., De Waele, J. J., \& Roberts, J. A. (2020). Antimicrobial therapeutic drug monitoring in critically ill adult patients: a Position Paper. Intensive Care Medicine, 46(6), 1127-1153. https://doi.org/10.1007/s00134-020-06050-1

Alp, E., \& Damani, N. (2015). Review Healthcare-associated infections in Intensive Care Units: epidemiology and infection control in low-to-middle income countries. The Journal of Infection in Developing Countries, 9(10), 1040-1045. https://doi.org/10.3855/jidc.6832

Alyahawi, A., Alrubaiee, G., \& Alkaf, A. (2020). Evaluation of Carbapenem Use Among Patients At Intensive Care Unit (Icu) in Sana'a, Yemen. Universal Journal of Pharmaceutical Research, 4(6), 30-34. https://doi.org/10.22270/ujpr.v4i6.333

Araujo, T. G. de, Rieder, M. de M., Kutchak, F. M., \& Franco Filho, J. W. (2013). Readmissões e óbitos após a alta da UTI - um desafio da terapia intensiva. Revista Brasileira de Terapia Intensiva Intensiva, 25(1), 32-38.

Balkhy, H. H., El-Saed, A., El-Metwally, A., Arabi, Y. M., Aljohany, S. M., Al Zaibag, M., Baharoon, S., \& Alothman, A. F. (2018). Antimicrobial consumption in five adult intensive care units: A 33-month surveillance study. Antimicrobial Resistance and Infection Control, 7(1), 1-9. https://doi.org/10.1186/s13756-018-0451-9

Baroni, F. C. de S., Ferreira, M. M., \& Roese, F. M. (2017). Perfil de utilização de antimicrobianos na unidade de terapia intensiva de um hospital público. Revista Brasileira de Farmácia Hospitalar e Serviços de Saúde, 8(4), 37-44. https://doi.org/10.30968/rbfhss.2017.084.007

Brasil. (2017). Diretriz Nacional para Elaboração de Programa de Gerenciamento do Uso de Antimicrobianos em Serviços de Saúde - Agência Nacional de Vigilância Sanitária. https://www20.anvisa.gov.br/segurancadopaciente/index.php/publicacoes/item/diretriz-nacional-para-elaboracao-de-programa-degerenciamento-do-uso-de-antimicrobianos-em-servicos-de-saude

Carvalho, P. P., Porto, M. de J., Barros, J. F., Magalhães, A. S., Nunes, L. S., Silva, I. C., Abreu, I. L. S. de, Souza, G. N., Neto, J. M. de R., \& Mendes, T. da S. (2021). Avaliação de pacientes críticos com suspeita de sepse em um hospital universitário. Research, Society and Development, 10(3), 1-12. https://doi.org/http://dx.doi.org/10.33448/rsd-v10i3.13570

Chatzopoulou, M., \& Reynolds, L. (2020). Role of antimicrobial restrictions in bacterial resistance control: a systematic literature review. Journal of Hospital Infection, 104(2), 125-136. https://doi.org/10.1016/j.jhin.2019.09.011

Codjoe, F. S., \& Donkor, E. S. (2018). Carbapenem Resistance : A Review. Medical Sciences, 6(1), 1-28. https://doi.org/10.3390/medsci6010001

Coêlho, T. da S., Oliveira, R. A., Lúcio Neto, M. P., Santos, P. N., \& Silva, G. de A. (2020). Análise do consumo de antimicrobianos em pacientes críticos de um hospital de referência no Piauí. Research, Society and Development, 9(7), 1-15. 
Furtado, D. M. F., Silveira, V. S. da, Carneiro, I. C. do R. S., Furtado, D. M. F., \& Kilishek, M. P. (2019). Consumo de antimicrobianos e o impacto na resistência bacteriana em um hospital público do estado do Pará, Brasil, de 2012 a 2016. Revista Pan-Amazônica de Saúde, 10(0), 1-8. https://doi.org/10.5123/s2176-6223201900041

Gajdács, M. (2019). The Concept of an Ideal Antibiotic: Implications for Drug Design. Molecules, 24, 1-16. https://doi.org/10.3390/molecules24050892

Joseph, N. M., Bhanupriya, B., Shewade, D. G., \& Harish, B. N. (2015). Relationship between antimicrobial consumption and the incidence of antimicrobial resistance in Escherichia coli and Klebsiella pneumoniae isolates. Journal of Clinical and Diagnostic Research, 9(2), DC08-DC12. https://doi.org/10.7860/JCDR/2015/11029.5537

Khan, H. A., Baig, F. K., \& Mehboob, R. (2017). Nosocomial infections: Epidemiology, prevention, control and surveillance. Asian Pacific Journal of Tropical Biomedicine, 7(5), 478-482. https://doi.org/10.1016/j.apjtb.2017.01.019

Kollef, M. H., Bassetti, M., Francois, B., Burnham, J., Dimopoulos, G., Garnacho-Montero, J., Lipman, J., Luyt, C.-E., Nicolau, D. P., Postma, M. J., Torres, A., Welte, T., \& Wunderink, R. G. (2017). The intensive care medicine research agenda on multidrug- resistant bacteria, antibiotics, and stewardship. Intensive Care Medicine, 43(9), 1187-1197. https://doi.org/10.1007/s00134-017-4682-7

Li, Y., Wang, J., Wang, R., \& Cai, Y. (2020). Double-carbapenem therapy in the treatment of multidrug resistant Gram- negative bacterial infections : a systematic review and meta-analysis. BMC Infectious Diseases, 20, 1-13.

Lima, A. L. L. M., Oliveira, P. R. D. de, Paula, A. P. de, Dal-Paz, K., Almeida Jr, J. N. de, Félix, C. da S., \& Rossi, F. (2011). Carbapenem stewardship: positive impact on hospital ecology. Brazilian Journal of Infectious Diseases, 15(1), 1-5. https://doi.org/10.1590/s1413-86702011000100001

Lobo, L. G., Ramos, F., Moretti, M. M., \& Alves, P. H. (2017). Resultados de um Programa Multidisciplinar de Otimização do Uso de Antimicrobianos. Revista de Epidemiologia e Controle de Infecção, 7(1), 1-5. https://doi.org/10.17058/reci.v7i1.7279

McCann, E., Srinivasan, A., Andrew DeRyke, C., Ye, G., DePestel, D. D., Murray, J., \& Gupta, V. (2018). Carbapenem-nonsusceptible Gram-negative pathogens in ICU and non-ICU settings in US hospitals in 2017: A multicenter study. Open Forum Infectious Diseases, 5(10), 1-7. https://doi.org/10.1093/ofid/ofy241

Micek, S. T., Welch, E. C., Khan, J., Pervez, M., Doherty, J. A., Reichley, R. M., \& Kollef, M. H. (2010). Empiric combination antibiotic therapy is associated with improved outcome against sepsis due to gram-negative bacteria: A retrospective analysis. Antimicrobial Agents and Chemotherapy, 54(5), 1742-1748. https://doi.org/10.1128/AAC.01365-09

O’Neill, J. (2014). Review on Antimicrobial Resistance. Antimicrobial Resistance: Tackling a crisis for the health and wealth of nations.

Oliva, A., Scorzolini, L., Castaldi, D., Gizzi, F., Angelis, M. De, Storto, M., D’Abramo, A., Aloj, F., Mascellino, M. T., Mastroianni, C. M., \& Vullo, V. (2017). Double-carbapenem regimen, alone or in combination with colistin, in the treatment of infections caused by carbapenem- resistant Klebsiella pneumoniae (CR-Kp). Journal of Infection, 74, 103-106. https://doi.org/10.1016/j.jinf.2016.10.002

Organização Mundial da Saúde. (2020). 2020 Antibacterial agents in clinical and preclinical development: an overview and analysis. https://www.who.int/publications/i/item/9789240021303

Organização Mundial da Saúde. (2021). WHO Collaborating Centre for Drug Statistics Methodology, Guidelines for ATC classification and DDD assignment 2021. https://www.whocc.no/filearchive/publications/2021_guidelines_web.pdf

Paiva, J. A., Mergulhão, P., \& Figueira Salluh, J. I. (2020). What every intensivist must know about antimicrobial stewardship: Its pitfalls and its challenges. Revista Brasileira de Terapia Intensiva, 32(2), 207-212. https://doi.org/10.5935/0103-507X.20200037

Pascale, G. De, Martucci, G., Montini, L., Panarello, G., Cutuli, S. L., Carlo, D. Di, Gravio, V. Di, Stefano, R. Di, Capitanio, G., Vallecoccia, M. S., Polidori, P., Spanu, T., Arcadipane, A., \& Antonelli, M. (2017). Double carbapenem as a rescue strategy for the treatment of severe carbapenemase-producing Klebsiella pneumoniae infections : a two-center , matched case - control study. Critical Care, 21, 1-10. https://doi.org/10.1186/s13054-017-1769-z

Patrier, J., \& Timsit, J. F. (2020). Carbapenem use in critically ill patients. Current Opinion in Infectious Diseases, 33(1), 86-91. https://doi.org/10.1097/QCO.0000000000000622

Roberts, J. A., Paul, S. K., Akova, M., Bassetti, M., Waele, J. J. De, Dimopoulos, G., Kaukonen, K., Koulenti, D., Martin, C., Montravers, P., Rello, J., Rhodes, A., Starr, T., Wallis, S. C., \& Lipman, J. (2014). DALI: Defining Antibiotic Levels in Intensive Antibiotic Doses Sufficient for Critically Ill Care Unit Patients: Are Current $\beta$-Lactam Patients? Clinical Infectious Diseases, 58(8), 1072-1083. https://doi.org/10.1093/cid/ciu027

Rodrigues, F. d'Athayde, \& Bertoldi, A. D. (2010). Perfil da utilização de antimicrobianos em um hospital privado. Ciência \& Saúde Coletiva, 15(suppl 1), 1239-1247. https://doi.org/10.1590/s1413-81232010000700033

Santos, P. N. do, Silva, G. de A., Coêlho, T. da S., Oliveira, R. A. de, \& Neto, M. P. L. (2020). Análise farmacoeconômica dos antimicrobianos na unidade de terapia intensiva em um hospital terciário. Research, Society and Development, 9(5), 1-18. https://doi.org/http://dx.doi.org/10.33448/rsd-v9i5.3179

Silva, M. C. M. da, Sousa, R. M. C., \& Padilha, K. G. (2011). Fatores associados ao óbito e a readmissão em Unidade de Terapia Intensiva. Revista LatinoAmericana de Enfermagem, 19(4), 1-9.

Souza, G. N., Porto, M. de J., Santos, J. P. dos, Freitas, A. L., \& Carvalho, P. P. (2021). Perfil das prescrições de antimicrobianos de uso restrito em uma Unidade de Terapia Intensiva. Research, Society and Development, 10(8), e15710816565. https://doi.org/10.33448/rsd-v10i8.16565

Wagner, J. L., Rhodes, N. J., Scheetz, M. H., Bosso, J. A., Goff, D. A., Rybak, M. J., \& Davis, S. L. (2020). Opportunities for antimicrobial stewardship among carbapenem-treated patients in 18 North American hospitals. International Journal of Antimicrobial Agents, 55(6), 105970. https://doi.org/10.1016/j.ijantimicag.2020.105970

White, B. P., Patel, S., Tsui, J., \& Chastain, D. B. (2019). Adding double carbapenem therapy to the armamentarium against carbapenem-resistant Enterobacteriaceae bloodstream infections. Infectious Diseases, 51(3), 161-167. https://doi.org/10.1080/23744235.2018.1527470 